\title{
Signal-on photoelectrochemical immunoassay for salivary cortisol based on silver nanoclusters-triggered ion-exchange reaction with CdS quantum dots
}

\author{
Dajuan Luo ${ }^{1}$ Q Qiuping $\mathrm{Fu}^{2} \cdot$ Rong Gao ${ }^{1} \cdot$ Lixia Su$^{1} \cdot$ Yonghuan Su${ }^{1} \cdot$ Bingqian Liu ${ }^{1}$
}

Received: 11 November 2021 / Revised: 20 December 2021 / Accepted: 10 January 2022 / Published online: 21 February 2022

(c) Springer-Verlag GmbH Germany, part of Springer Nature 2022

\begin{abstract}
Nowadays, the epidemic, employment, and academic pressures are seriously affecting our physical and mental health. Herein, we designed a magneto-controlled photoelectrochemical immunosensor for noninvasive monitoring of salivary cortisol regarded as a pressure biomarker. A competitive immunoassay model was established by coupling bovine serum albumincortisol modified magnetic beads (MB-BSA-cortisol) with silver nanoclusters (Ag NCs)-labelled anti-cortisol antibody, and quantity analysis was operated by photoelectrochemical measurement of the CdS/Au electrode as an ion-exchange platform. Accompanying the formation of immune complexes, the carried Ag NCs were readily dissolved with nitric acid to produce abundant silver ions, which transferred to the electrode for ion-exchange reaction with CdS quantum dots to produce $\mathrm{Ag}_{2} \mathrm{~S}$, a new electron-hole capture site, leading to a decrease in the photocurrent intensity. The photocurrent signal gradually recovered with the increase of concentration of target cortisol, acquiring the signal-on mode competitive immunosensing system, which is propitious to the detection of small molecules. Within optimal conditions, this sensor had a satisfactory linear relationship in the range of $0.0001-100 \mathrm{ng} \mathrm{mL}^{-1}$ with favorable repeatability, specificity, and acceptable method accuracy. The detection limit was as low as $0.06 \mathrm{pg} \mathrm{mL}^{-1}$. In addition, this strategy provided new thought for the test of other small-molecule analytes and immunosensor applied in the complex biological system.
\end{abstract}

Keywords Photoelectrochemical $\cdot$ Competitive immunoassay $\cdot$ Ion-exchange reaction $\cdot$ Small-molecule cortisol

\section{Introduction}

During the current coronavirus pandemic, it has been reported that discussion of stress etiology is going on increasingly among health care workers as well as the general public [1-3]. Apparently, developing a simple and sensitive method for the detection of stress biomarkers is of great significance in health protection. Cortisol, a biomarker of stress detection in humans considered commonly, in particular, salivary cortisol, has become highly attractive compared to the assays in blood, sweat, or hair due to simple,

Bingqian Liu

nqliu@gzu.edu.cn; bingqianliu_gzu@163.com

1 Guizhou Engineering Laboratory for Synthetic Drugs (Ministry of Education of Guizhou Province), College of Pharmacy, Guizhou University, Guiyang 550025, China

2 College of Chemistry and Materials Engineering, Guiyang University, Guiyang 550005, China noninvasive, and well reproducible sampling [4]. Photoelectrochemical (PEC) sensing has recently gained more and more popularity in the fields of biological and chemical analysis [5, 6], which incorporates the excellent merits of photochemical method and electrochemical technology [7], including rapid detection, low cost, good sensitivity, and wide linearity range [8]. Inspiringly, PEC sensing, in the noninvasive monitoring of small molecules in biological fluids, has been reported in succession and demonstrated tremendous progress [9-11]. However, research on the PEC assay of salivary cortisol is still lacking now.

It is well-known that signal-on biosensors are superior to signal-off ones because of their lower background signal and probability for false-positive results $[12,13]$. What's more, in PEC immunoassay, the quantitative analysis of biomarkers is usually carried out through photocurrent changes after biological recognition. Nevertheless, some of the analytes tend to be low in the early stages of diseases. Therefore, it is considerable to employ signal amplification strategies into the PEC system [14-16]. To date, multifarious 
Scheme 1 Schematic illustration of the as-designed PEC biosensor for cortisol in saliva
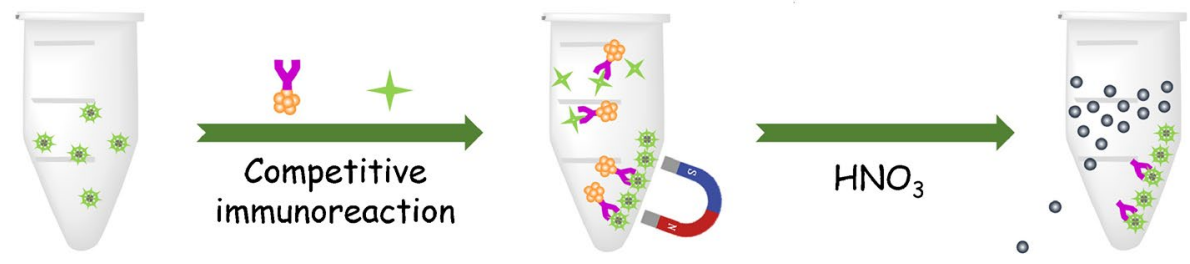

$\circ$
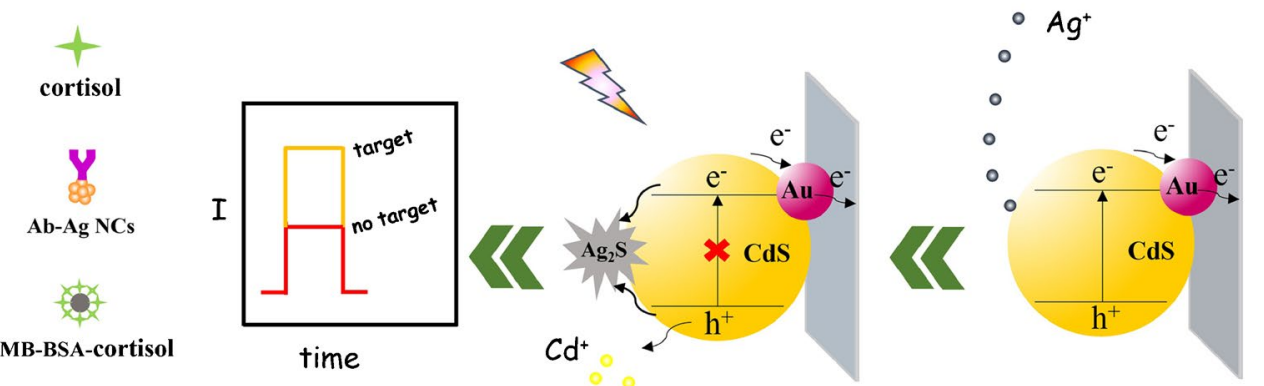

signal amplification strategies have been put forward for PEC immunoassay to enhance sensitivity, such as enzymemediated catalytic [17], energy transfer [18], chemical redox cycling [19], nano-material labels [20], and other strategies. Among them, ion-exchange technology has opened new roads for designing immune sensors in recent years because of its rapid reaction with another element at room temperature to form a new photoactive species to amplify the change in photocurrent value $[21,22]$. Zhang et al. established a PEC sensing system based on an ion-exchange reaction between copper ions $\left(\mathrm{Cu}^{2+}\right)$ and $\mathrm{WO}_{3}-\mathrm{Au}-\mathrm{CdS}$ nanoparticles, forming the weak photoactive material $\mathrm{Cu}_{\mathrm{x}} \mathrm{S}$ to reduce the photocurrent [23]. Song et al. constructed an excellent biosensor for microRNA detection through the ion-exchange reaction between silver ions and $\mathrm{CdSe}$ QDs/TiO 2 composites [24]. Likewise, Zhao et al. proposed a PEC platform via the silver nanoclusters-based ion-exchange reaction with $\mathrm{CdTe}$ QDs, which dramatically enlarged the PEC response for ultrasensitive target detection [25]. Therefore, PEC immunosensors based on ion exchange have great potential in biological analysis in the future.

Since the photocurrent signal arose from the charge separation and migration upon illumination between photoactive material and electrode $[8,26]$, the active materials play a crucial role in the PEC sensing system. With the continuous development of semiconductor nano-material, they are increasingly widely used in PEC fields, particularly quantum dots (QDs), attributed to their efficient photoelectric conversion, desirable optoelectronic property, adjustable band gap, and unique biocompatibility [27-29]. CdS, an n-type semiconductor with excellent visible light activity, has become one of the intensively studied photoelectric materials, yet pure CdS also suffers from severe photo-corrosion [30]. Fortunately, this drawback can be overcome by doping $\mathrm{Au}$ nanoparticles (NPs) into CdS due to the surface plasmon resonance (SPR) of Au NPs, which acts as electron transfer mediator, effectively separates and transfers photogenerated charge carriers [31-33].

Consequently, our motivation is to develop a novel PEC immunosensing platform for small-molecular cortisol in salivary via the ion-exchange reaction between $\mathrm{Ag}$ NCs as the labels and Au NPs decorated CdS quantum dots as photoactive receptors. As shown in Scheme 1, this assay was a competitive-immune and split-type detection mode. In a centrifugal tube, bovine serum albumin-cortisol modified with magnetic meads (MB-BSA-cortisol) were used as competitors to compete with the target cortisol for silver nanoclusters labelled anti-cortisol antibody (Ab-Ag NCs). Then, magnetic separation removed both the $\mathrm{Ab}-\mathrm{Ag} \mathrm{NCs}$ bound target and the unbound target cortisol. Subsequently, the carried Ag NCs onto magnetic beads accompanying the antigen-antibody reaction was expected to release numerous $\mathrm{Ag}^{+}$with the aid of nitric acid $\left(\mathrm{HNO}_{3}\right)$. Finally, the as-released $\mathrm{Ag}^{+}$ could exchange with $\mathrm{CdS}$ QDs to form $\mathrm{Ag}_{2} \mathrm{~S}$ nanocrystals, which mediated the exciton trapping and resulted in a remarkable reduction in the photocurrent of the $\mathrm{CdS} / \mathrm{Au}$ photoelectrode. The above ion-exchange reaction: $[(\mathrm{CdS}) n+$ $\left.2 \mathrm{n} \bullet \mathrm{Ag}^{2+}(\mathrm{aq}) \rightarrow\left(\mathrm{Ag}_{2} \mathrm{~S}\right) n+\mathrm{n} \bullet \mathrm{Cd}^{2+}(\mathrm{aq})\right]$ was feasible at room temperature, accompanied by the color changing from yellow to dark brown, which was on account of the fact that the solubility product constant of $\mathrm{Ag}_{2} \mathrm{~S}\left(K_{\mathrm{sp}} \sim 6.69 \times 10^{-50}\right)$ was lower than that of $\mathrm{CdS}\left(8.00 \times 10^{-27}\right)$, rendering fractional replacement of $\mathrm{Cd}^{2+}$ ions with $\mathrm{Ag}^{+}$ions [34]. MBBSA-cortisol not only served as competitors to participate in competitive immunoassay that was recognized as the preferred method for small-molecule immunoassay but also facilitated excluding coexisting species from actual samples under external magnetic fields to enhance specificity. Also, as Ag NCs could release a large number of silver ions for ion exchange compared with silver nanoparticles and achieve 
double signal amplification, the sensitivity of such a PEC biosensor is doomed to be preferable. As expected, the asdesigned PEC sensors were capable of monitoring cortisol in saliva samples, and its photocurrent signal is proportional to the target cortisol level with excellent selectivity and sensitivity. Hence, it had potential applications in stress testing.

\section{Experimental section}

\section{Chemicals and materials}

Anti-cortisol mouse monoclonal antibody (XM210, Ab) was ordered from Abcam (England). Thioctic acid $(99 \%, \mathrm{~m} / \mathrm{m})$, Cadmium chloride hemi (pentahydrate) $\left(\mathrm{CdCl}_{2} \cdot 2.5 \mathrm{H}_{2} \mathrm{O}\right.$, $98 \%, \mathrm{~m} / \mathrm{m}$ ), hydrocortisone (cortisol, $98 \%, \mathrm{~m} / \mathrm{m}$ ), and progesterone (Pro, $98 \%, \mathrm{~m} / \mathrm{m}$ ) were all obtained from Shanghai Macklin Biochemical Co., Ltd. (China). 1-ethyl-3-(3-dimethyl-aminopropyl) carbodiimide hydrochloride (EDC, 99\%, $\mathrm{m} / \mathrm{m}$ ) was purchased from Shanghai Yuanye Bio-Technology Co., Ltd. (China). N-hydroxysuccinimide (NHS, 99.0\%, $\mathrm{m} / \mathrm{m}$ ) and bovine serum albumin (BSA) derived from Beijing Solarbio Science Technology Co., Ltd. (China). Silver nitrate $\left(\mathrm{AgNO}_{3}, 99.8 \%, \mathrm{~m} / \mathrm{m}\right)$, sodium borohydride $\left(\mathrm{NaBH}_{4}, 96.0 \%\right.$, $\mathrm{m} / \mathrm{m})$, iron trichloride hexahydrate $\left(\mathrm{FeCl}_{3} \cdot 6 \mathrm{H}_{2} \mathrm{O}, 99 \%, \mathrm{~m} / \mathrm{m}\right)$, and tetrachloroauric (III) acid tetrahydrate $\left(\mathrm{HAuCl}_{4} \cdot 4 \mathrm{H}_{2} \mathrm{O}\right.$, $47.8 \%, \mathrm{~m} / \mathrm{m}$ ) were all obtained from Sinopharm Chemical Reagent Co., Ltd. (Shanghai, China). Glucose (Glu) was obtained from Chengdu Jinshan Chemical Test Co., Ltd. (China). Sodium sulfide nonahydrate $\left(\mathrm{Na}_{2} \mathrm{~S} \cdot 9 \mathrm{H}_{2} \mathrm{O}, 98.0 \%\right.$, $\mathrm{m} / \mathrm{m}$ ) was obtained from Xilong Scientific Co., Ltd. Nitric acid $\left(\mathrm{HNO}_{3}, 65.0-68.0 \%\right.$, v/v) was purchased from Chongqing Chuandong Chemical Co., Ltd. (China). Phosphate buffer solution (PBS) was prepared with the following reagents: sodium phosphate dibasic $\left(\mathrm{Na}_{2} \mathrm{HPO}_{4}, 99.0 \%, \mathrm{~m} / \mathrm{m}\right)$ and sodium dihydrogen phosphate $\left(\mathrm{NaH}_{2} \mathrm{PO}_{4}\right)$ with monitoring by pHST-310 Ohaus $\mathrm{pH}$ meter (USA), which was calibrated via standard $\mathrm{pH}$ buffer solutions. Ultrapure water used in all solutions was from tap water purified by a Water Milli-Q system (Merck KGaA, Germany). Human salivary cortisol enzyme-linked immunosorbent assay (ELISA) kit (Cat. No. RXJ103617H; inter-assay CV $<15 \%$; intra-assay $\mathrm{CV}<15 \%$; sensitivity: $0-0.036 \mathrm{ng} \mathrm{mL}^{-1}$; examination area: 1.1-17.4 $\mathrm{ng} \mathrm{mL}^{-1}$ ) was purchased from Ruixin Biotech (China).

\section{Apparatus}

X-ray diffraction (XRD) analysis was implemented on a Shimadzu XRD-6000 diffractometer (Shimadzu, Shimadzu, Japan). High resolution transmission electron microscopy (HRTEM) characterization was completed on a JEM-2100F microscope (JEOL, Tokyo, Japan). The characterization of elements was carried out by a Thermo Scientific Escalab 250Xi X-ray photoelectron spectroscopy (Thermo, MMAS, USA). The UV-vis absorption spectra were tested on a UV5500PC UV-visible spectrophotometer (Metash, Shanghai, China). F-4700 spectrophotometer was used to perform the fluorescence spectra (Hitachi, Tokyo, Japan). The zeta potential was estimated by NanoBrook Omni Brookhaven Instruments (BIC, Brookhaven, USA). The FT-IR characteristic measurement was carried out by L1600400 Spectrum Two infrared spectrometer (PerkinEImer, LIantrisant, UK). Electrochemical impedance spectroscopy (EIS) and cyclic voltammetry (CV) were carried out at the CHI 660E electrochemical workstation (Shanghai Chenhua Instrument, Shanghai, China).

\section{Preparation of Ab-Ag NCs and MB-BSA-cortisol}

The synthesis process of Ag NCs and MB was described in the electronic supplementary information (ESM). Like the reported method, the as-prepared $\mathrm{Ag} \mathrm{NC}$ was used to label anti-cortisol antibodies [35]. First, $1 \mathrm{~mL}$ Tris-EDTA buffer solution contained $3 \mathrm{mM}$ glutathione was added to a brown Eppendorf tube, subsequently joining anti-cortisol antibody $(100 \mu \mathrm{L}, 100 \mu \mathrm{g} / \mathrm{mL})$ together with $188 \mu \mathrm{L}$ of $\mathrm{Ag}$ NCs and $1 \mathrm{~mL}$ solution containing EDC and NHS (0.01 M). The above solution was mixed evenly on the vortex apparatus and then shaken gently on a shaker for $2 \mathrm{~h}$ at room temperature. Finally, purification (100 kDa pore size, Amicon ultra centrifugal filter units) was performed using the following process: a first cycle of $5500 \mathrm{rpm}$ for $10 \mathrm{~min}$ and two ultrapure water cleaning cycles at $5500 \mathrm{rpm}$ for $5 \mathrm{~min}$. The Ag NCs-labelled anti-cortisol antibody (Ab-Ag NCs) in the filter was dispersed in PBS $(2 \mathrm{~mL}, 0.1 \mathrm{M}, \mathrm{pH}=7.4)$ and was stored in the fridge.

According to the previous method [36], the magnetic beads need to carboxylate before biological coupling. In the first, $1.0 \mathrm{~g}$ of bare magnetic beads was added to a roundbottomed flask containing $100 \mathrm{~mL}$ ultrapure water followed by ultrasonic dispersion. Then, under nitrogen protection, $2.5 \mathrm{~g}$ of citric acid was dissolved in the suspension and kept for $30 \mathrm{~min}$, and heated the suspension to $70{ }^{\circ} \mathrm{C}$ for refluxing $1 \mathrm{~h}$. At the end, using a permanent magnet to collect carboxylated magnetic beads after thorough washing with ultrapure water, dried in a vacuum. The carboxylated magnetic beads and BSA-cortisol were conjugated by a classical carbodiimide coupling referring to the previous report with slight modifications [11]. In brief, $1.0 \mathrm{~mL}$ of the carboxylated magnetic beads $(5.0 \mathrm{mg} / \mathrm{mL})$ was dispersed in $1.0 \mathrm{~mL}$ PBS solution (0.1 M, pH 7.4) with $100 \mathrm{mM}$ NHS and $400 \mathrm{mM}$ EDC. The resulting solution was then lightly shaken for $60 \mathrm{~min}$ before being magnetically separated and washed three times with ultrapure water to take out superfluous EDC and NHS. Afterwards, the active magnetic beads 
were dispersed in a BSA-cortisol solution $\left(5 \mathrm{mg} \mathrm{mL}^{-1}\right)$ and shaken gently overnight. Finally, the obtained BSA-cortisol modified with magnetic meads (MB-BSA-cortisol) was dispersed in $2.0 \mathrm{~mL}$ PBS and stored in the refrigerator.

\section{Photoelectrode preparation and PEC immunoassay}

The synthesis process of CdS QDs and Au NPs is shown in ESM. The fluorine-tin-oxide electrode (FTO) electrode was cleaned by sonication with acetone, ethanol, and ultrapure water in sequence and dried at $60{ }^{\circ} \mathrm{C}$ before use. Then, the waterproof transparent tape punched with a hole ( $r=0.28 \mathrm{~cm}, S=0.25 \mathrm{~cm}^{2}$ ) was stuck on the surface of the electrode. After that, $30 \mu \mathrm{L}$ of Au NPs colloids was dropped into the hole of the FTO electrode. After drying naturally at room temperature, $20 \mu \mathrm{L}$ CdS QDs solution was also coated on the surface of the above electrode and dried at $40{ }^{\circ} \mathrm{C}$.

Scheme 1 describes the schematic illustration of the PEC sensing system for target cortisol based on Ag NCs-aided ion-exchange reaction with $\mathrm{CdS}$ quantum dots. The competitive reaction was carried out in a $500 \mu \mathrm{L}$ centrifugal tube containing $100 \mu \mathrm{L}$ of the as-synthesized MB-BSA-cortisol. First, $50 \mu \mathrm{L}$ of target cortisol standard/sample together with $50 \mu \mathrm{L}$ of Ab-Ag NCs (certain concentration as prepared) were injected into the tube and incubated at $37^{\circ} \mathrm{C}$ for $50 \mathrm{~min}$ on a shaker. The resulting suspension was magnetically separated and washed twice with PBS $(0.01 \mathrm{M}, \mathrm{pH}=7.4)$ to remove unbound antibodies and target cortisol. Following that, $30 \mu \mathrm{L} \mathrm{HNO}_{3}(0.1 \mathrm{mM})$ was added into the tube and reacted for $15 \mathrm{~min}$ to release abundant $\mathrm{Ag}^{+}$from the competed Ag NCs labels. Afterwards, with the aid of magnetic, $20 \mu \mathrm{L}$ of supernatant droplet containing $\mathrm{Ag}^{+}$was deposited on the CdS/Au-modified FTO electrode, then kept at room temperature for $20 \mathrm{~min}$ to execute the ion-exchange between $\mathrm{Ag}^{+}$and CdS QDs. At last, the resulting electrode was washed with ultrapure water for subsequent PEC detection. The photocurrent was tested in $0.1 \mathrm{M} \mathrm{Na}_{2} \mathrm{SO}_{4}$ solution $(10 \mathrm{~mL})$ with a traditional three-electrode system using the obtained electrode as the working electrode, a platinum wire electrode as the counter electrode, and a saturated $\mathrm{Ag} / \mathrm{AgCl}$ electrode as the reference electrode. The electrochemical workstation configured with a $500 \mathrm{~W}$ Mexe lamp (Beijing NBET Technology, China) as the excitation light source, which switched on and off every $10 \mathrm{~s}$.

\section{Results and discussion}

\section{Materials characterization}

In practical application, it was necessary to confirm whether the prepared CdS QDs can be exchanged by $\mathrm{Ag}^{+}$. For this purpose, we used different techniques to characterize $\mathrm{CdS}$
QDs before and after exchange with certain $\mathrm{Ag}^{+}$ions. Figure 1A showed a HRTEM image of the as-prepared CdS QDs with an average size of approximately $8 \mathrm{~nm}$. When CdS QDs reacted with excessive $\mathrm{Ag}^{+}$, we dimly saw from Fig. 1B that the shape and lattice of the resulting crystal were diverse those of alone CdS QDs (morphologies of other magnifications in Fig. S1). In addition, by comparing curve "black" vs curve "red" in Fig. 1C, we observed clearly the XPS characteristics of the Ag 3d double peak for the resulting $\mathrm{Ag}^{+}$, indicating that silver element exists in nanocrystals. For better understanding, we had also carried out the core-level spectra of $\mathrm{Cd}$ and $\mathrm{Ag}$, as exhibited in Fig. S3A and S3B. It was evident that the intensity of Cd $3 d_{5 / 2}$ and $3 d_{3 / 2}$ decreased, while it was seen that of $\mathrm{Ag}$ $3 d_{5 / 2}$ and $3 d_{3 / 2}$ increased correspondingly. Furthermore, the crystal structures of the materials successively modified on the electrodes were characterized by XRD in Fig. 1D. Except for the diffraction peaks of the FTO substrate, several typical characteristic peaks of CdS QDs located at $2 \theta=26.5^{\circ}$, $43.8^{\circ}$, and $51.8^{\circ}$ were indexed to the (111), (220), and (311) crystal planes, which coincided with the phase of CdS QDs (JCPDS 80-0019) [37]. And, the characteristic peak of $\mathrm{Ag}_{2} \mathrm{~S}$ generated by ion exchange on the electrode was not obvious, which could be caused by overlapping with that of CdS QDs. Nevertheless, it varied significantly at a peak magnification of about $37.5^{\circ}$ in Fig. S3C, as done that of the Au NPs.

The structural and optical characteristics of the prepared Ag NCs were characterized by UV-vis, fluorescence spectra, and TEM. Figure 2A demonstrated three characteristic absorption spectra of the Ag NCs (curve a), centered at 329, 427 , and $497 \mathrm{~nm}$ respectively which was in accordance with previous reports [38]. Then, the Ag NCs were functionalized with anti-cortisol antibodies to prepare signal nanoprobes and perform spectral characterization. As shown in Fig. 2A, the spectrum of Ab-Ag NCs (curve c) consisted of the characteristic peak of antibody at about $260 \mathrm{~nm}$ (curve b) and that of $\mathrm{Ag} \mathrm{NCs,} \mathrm{indicating} \mathrm{that} \mathrm{the} \mathrm{functionalization}$ was successful. Besides, from Fig. 2C, for zeta potential, the negative charges of $\mathrm{Ag} \mathrm{NCs}$ reduced after modification with $\mathrm{Ab}$, which also validated the successful conjugation of $\mathrm{Ab}$ onto Ag NCs. The emission spectra of the Ag NCs present a maximum of about $670 \mathrm{~nm}$ in Fig. 2B. However, the fluorescence characteristics disappeared under $\mathrm{HNO}_{3}$ treatment attributed to generating ions, which served as a source of $\mathrm{Ag}^{+}$for ion-exchange reactions. Moreover, the size and shape of Ag NCs were characterized through TEM. As illustrated in Fig. S2A, the Ag NCs were uniformly distributed with a mean diameter of $2-5 \mathrm{~nm}$ and could be seen a distinct lattice plane with the inter-fringe distance of $0.22 \mathrm{~nm}$. In addition, the biological coupling of magnetic beads is also characterized by UV-vis shown in Fig. 2D, and the MB-BSA-cortisol had both the absorption spectrum of MB and BSA-cortisol, demonstrating the successful fabrication. 

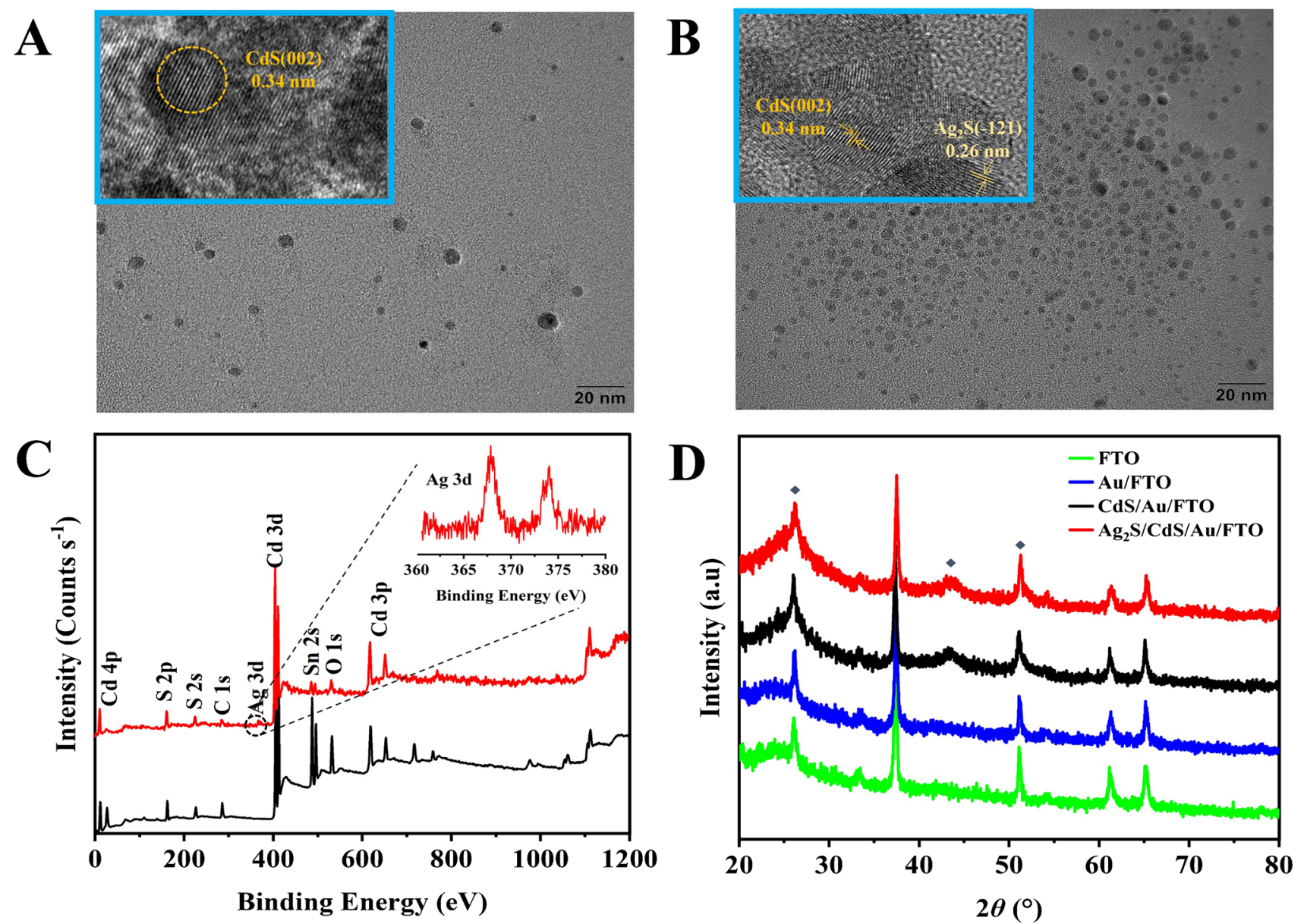

Fig. 1 HRTEM images of A CdS QDs and B CdS QDs + excess $\mathrm{Ag}^{+}$. Inset: magnification image; C XPS analyses of CdS QDs (black) and CdS QDs added excess $\mathrm{Ag}^{+}$(red). Inset: $\mathrm{Ag} 3 \mathrm{~d}$ core level XPS spectra; $\mathbf{D}$ the changes of XRD pattern on FTO electrode

Furthermore, the FT-IR spectra further confirmed this result, as shown in Fig. S2B, and the absorption peaks of the synthesized MB-COOH are consistent with the literature [39]. Compared with the MB-COOH, the MB-BSA-cortisol presented newly absorbance peaks located at $1540 \mathrm{~cm}^{-1}$ and $1630 \mathrm{~cm}^{-1}$, which are ascribed to $\mathrm{C}=\mathrm{C}$ bonds from aromatic rings of the cortisol molecule [40]. In addition, a broad peak of $-\mathrm{OH}$ at $3200 \mathrm{~cm}^{-1}$ shifted to lower wave numbers, which may be due to the hydrogen bond association between hydroxyl group on cortisol surface and water.

\section{Investigation of PEC performance and feasibility}

It is crucial to evaluate the PEC property of the prepared $\mathrm{CdS} / \mathrm{Au}$ electrode, which directly determines the monitoring performance of the analyte. As depicted in Fig. 3A, the photocurrent response of Au NPs modified electrode was very weak, almost the same as that of bare FTO electrode. After the assembly in turn of Au NPs and CdS QDs, the photocurrent intensity of $\mathrm{CdS} / \mathrm{Au}$, as expected, was much stronger than that of CdS alone; also, the photocurrent was stable and reliable (in Fig. S4A). The enhanced PEC response was mainly due to the modification of Au NPs on CdS QDs, which greatly promoted the separation and transfer of photogenerated electron-hole pair at the CdS/Au Schottky junction interface. EIS measurements were also conducted for the different modified FTO electrodes. In the Nyquist diagram, there are two main arcs in the high-frequency region (semicircle, $R_{\mathrm{ct}}$ ) and the low-frequency region (line segment, $R_{\mathrm{w}}$ ), respectively, representing the charge transport resistance and the recombination resistance, where the recombination resistance is inversely proportional to the recombination rate. As shown in Fig. 3B, it was clear that $R_{\mathrm{ct}}$ of $\mathrm{CdS} / \mathrm{Au}$ was much lower than that of $\mathrm{CdS}$ alone, while $R_{\mathrm{w}}$ was slightly higher than that of CdS, namely, the recombination rate of $\mathrm{CdS} / \mathrm{Au}$ electrode was lower. In addition, it also could be verified that the introduction of Au NPs could improve conductivity and promote electron transport from the CV curves in the inset. Taken together, all of the above results reveal the excellent PEC performance of the CdS/ 

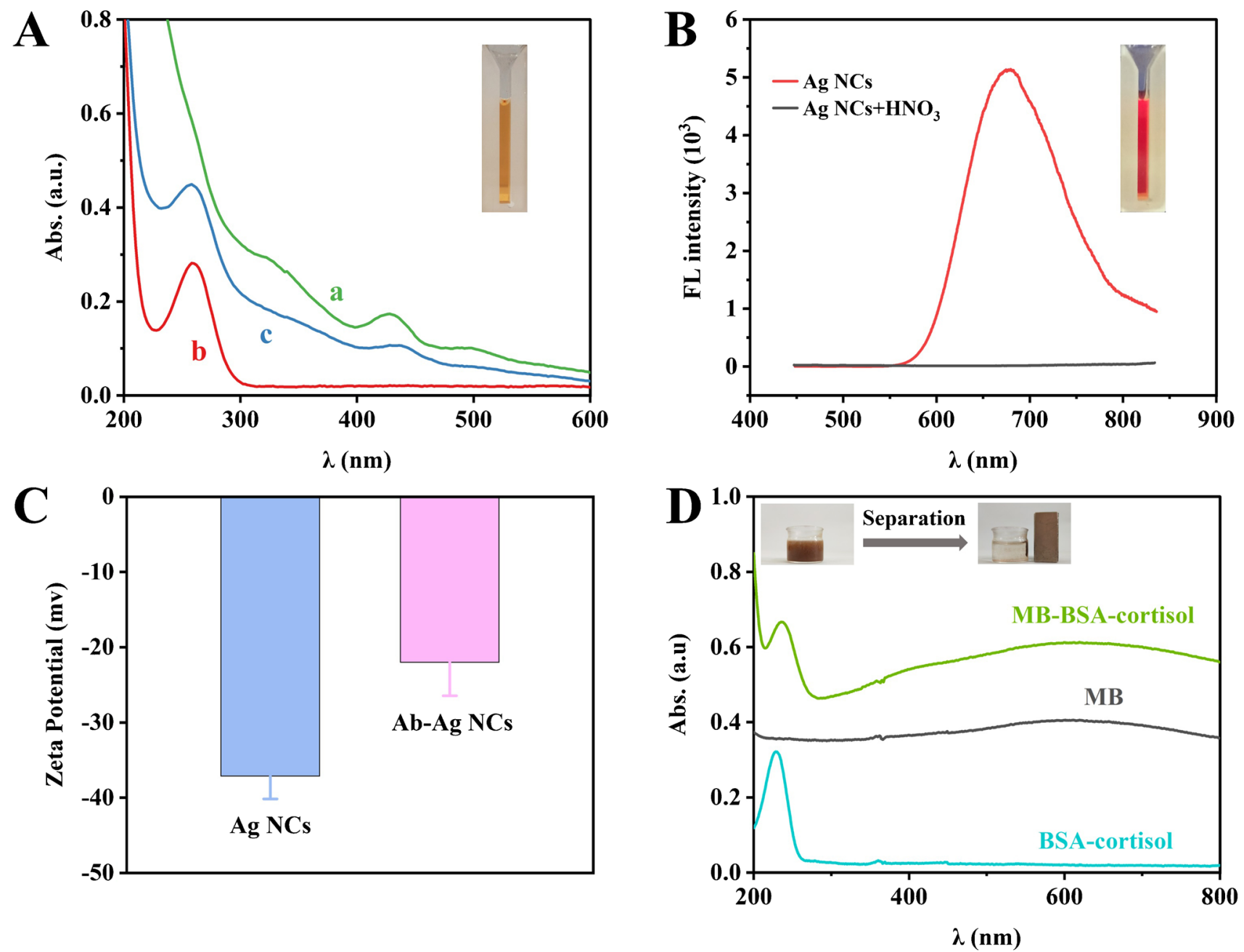

Fig. 2 A UV-vis absorption of (a) Ag NCs, (b) antibody, and (c) $\mathrm{Ab}-\mathrm{Ag} \mathrm{NCs}$. B The fluorescence of Ag NC disappeared after acid treatment. Inset: photos of the Ag NCs under visible light (A) and $365 \mathrm{~nm}$ UV light irradiation (B); $\mathbf{C}$ change of zeta potential after

$\mathrm{Au}$ electrode. In addition, we also performed the complete electrochemical characterizations of the sensor (please see ESM and Fig. S5).

Furthermore, when $\mathrm{Ag}^{+}$was introduced into the system, the photocurrent of the CdS/Au electrode decreased significantly than that of the pure CdS electrode (Fig. 3C, "II" vs "I"), accompanying different color changes in the inset, respectively. Significantly, the photocurrent signal decreases successively with the increase of $\mathrm{Ag}^{+}$concentration in Fig. S4B. According to the results obtained, it could be concluded that the CdS/Au electrode could be used to develop a competitive immunoassay using the Ag NCslabelled strategy. As demonstrated in Fig. 3D, curve "a" displayed the photocurrent response of CdS/Au-modified FTO. When the modified electrode was applied to the immune system, the photocurrent decreased significantly without target (curve "b"). In comparison, curve "c" exhibited the

modification of antibody onto Ag NCs. D the absorption spectra of MB, BSA-cortisol, and MB-BSA-cortisol (Inset: the superparamagnetic behavior of as-prepared MB under an external magnetic field)

photocurrent recovered with target cortisol $\left(1.0 \mathrm{ng} \mathrm{mL}^{-1}\right)$ intervention. The results revealed that silver ions produced by the immune complex after acid treatment resulted in a significant reduction of photocurrent signal; meanwhile, the photocurrent was proportional to the target concentration. In order to understand why the photocurrent decreased, we study the electronic band structures of CdS QDs by the Mott-Schottky (M-S) analyses in Fig. S6, where, please see ESI for detailed calculation of $E_{\mathrm{g}}, E_{\mathrm{cb}}$, and $E_{\mathrm{vb}}$ of CdS QDs. Notably, the positive slopes of the tangents line in the M-S plots for CdS suggested its intrinsic n-type semiconductor characteristics. According to the literature [41], the $\mathrm{Ag}_{2} \mathrm{~S}$ formed on the surface generated a new energy level with the band gap $\left(E_{\mathrm{g}}\right)$ of $0.92 \mathrm{eV}$, which was below that of CdS QD. Concretely, the conduction band $\left(E_{\mathrm{cb}},-0.52 \mathrm{eV}\right)$ of CdS was more catholic than that of $\mathrm{Ag}_{2} \mathrm{~S}(0 \mathrm{eV})$, while the valence band $\left(E_{\mathrm{vb}},+1.88 \mathrm{eV}\right)$ of $\mathrm{CdS}$ was more anodic than that of 

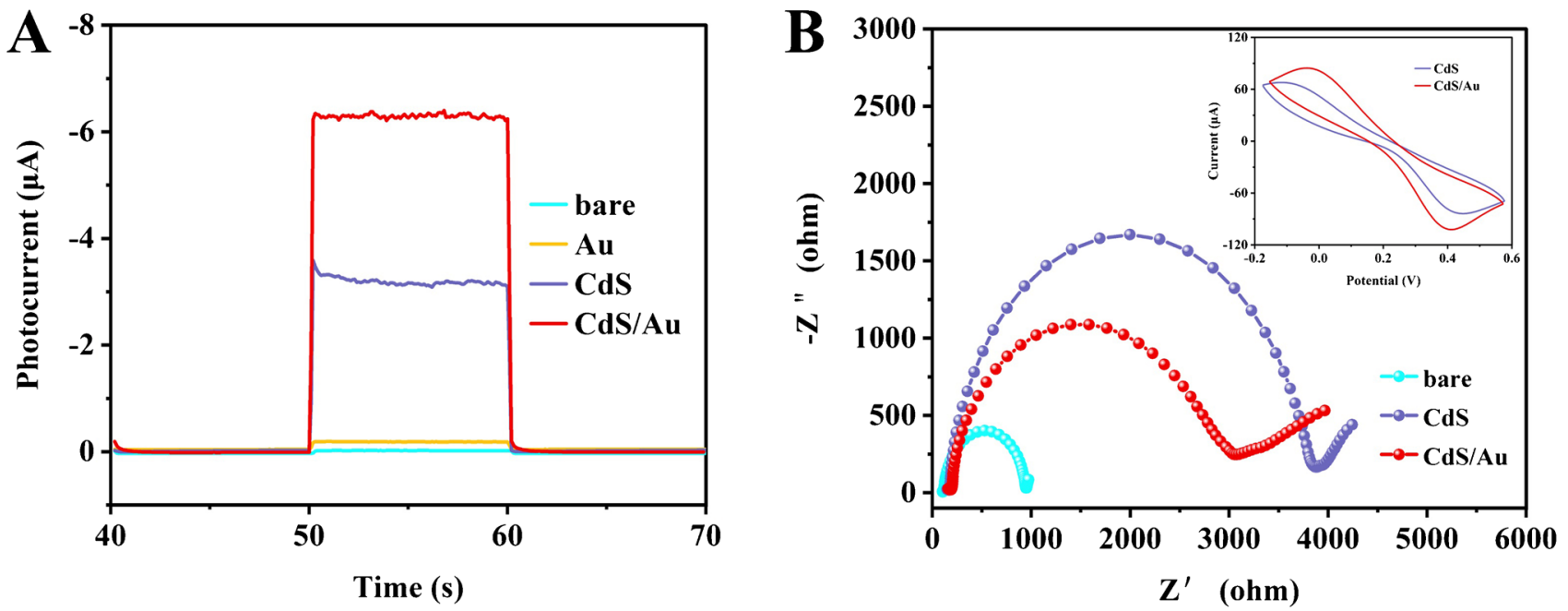

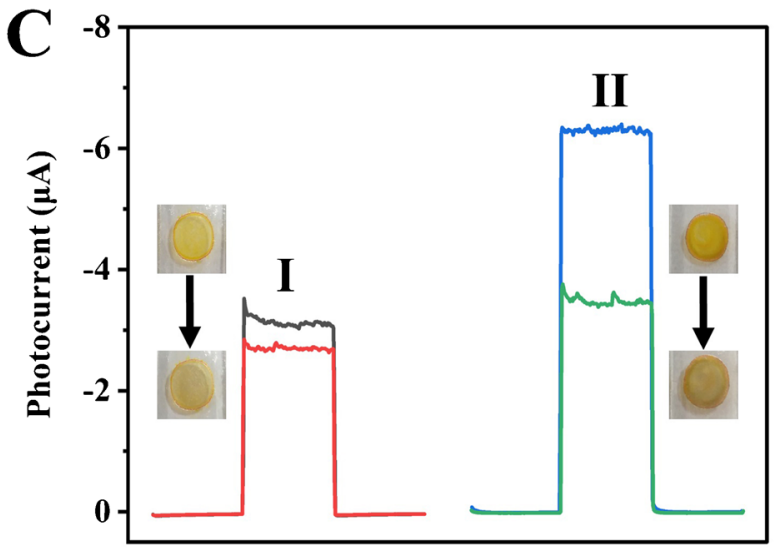

Time (s)

Fig. 3 A photocurrent responses. B Nyquist impedance plots (Inset: cyclic voltammetry curves). C Photocurrents of CdS QDs-modified electrode (I) and CdS/Au-modified electrode (II) in the absence (top)

$\mathrm{Ag}_{2} \mathrm{~S}(+0.92 \mathrm{eV})$, thus forming the trapping sites in Fig. S7. The above demonstrated that the signal-on and competitivetype PEC immunesensor was successfully constructed based on ion-exchange reaction on the $\mathrm{CdS} / \mathrm{Au}$ electrode.

\section{Analytical performance of PEC immunoassay}

To obtain the best performance of the PEC biosensor, it was necessary to optimize its potential, competitive immune time and ion-exchange reaction time. First, as indicated in Fig. S8A, a lower potential would minish the photocurrent of the CdS/Au electrode, while a higher one also did not significantly increase the photocurrent. Therefore, $0 \mathrm{~V}$ was selected for PEC measurement in this work. Next, as can be seen from Fig. S8B, with the extension of reaction time, the photocurrent gradually reduced, reaching a stable value at $50 \mathrm{~min}$ and no longer
D

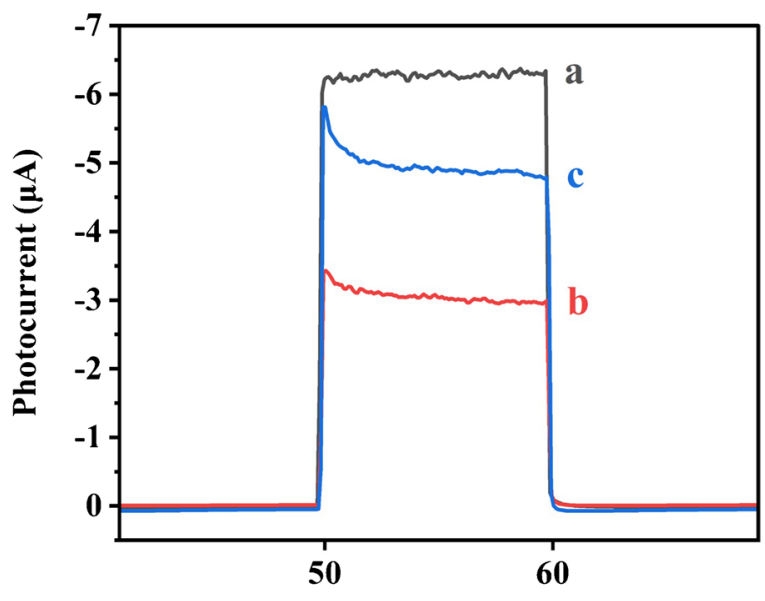

Time (s)

and presence (bottom) of $\mathrm{Ag}^{+}$ions; $\mathbf{D}$ photocurrent responses of the $\mathrm{CdS} / \mathrm{Au}$ electrode in the (a) background signal, (b) without, and (c) with of $1.0 \mathrm{ng} \mathrm{mL}^{-1}$ cortisol

decreasing. So, the reaction time of $50 \mathrm{~min}$ is the best time for a competitive immune system. Certainly, it also greatly influenced the photocurrent of the system in the ion-exchange time between CdS QDs and the immunoreaction product $\left(\mathrm{Ag}^{+}\right)$. As shown in Fig. S8C, with the increase of reaction time, the photocurrent of the $\mathrm{CdS} /$ $\mathrm{Au}$ electrode gradually decreased and levelled off after $15 \mathrm{~min}$. In order to minimize the determination time, it was appropriate that the ion-exchange reaction is $15 \mathrm{~min}$. Under the above best experimental parameters, the competitive-type immunosensing system was used for subsequent detection. Figure 4A presents the photocurrents of the PEC biosensor toward different concentrations of the target cortisol. It was observed that the photocurrent signals gradually increased with increasing cortisol concentration. As observed from Fig. 4B, the photocurrent had a well-fitted linear dependence with the logarithm 

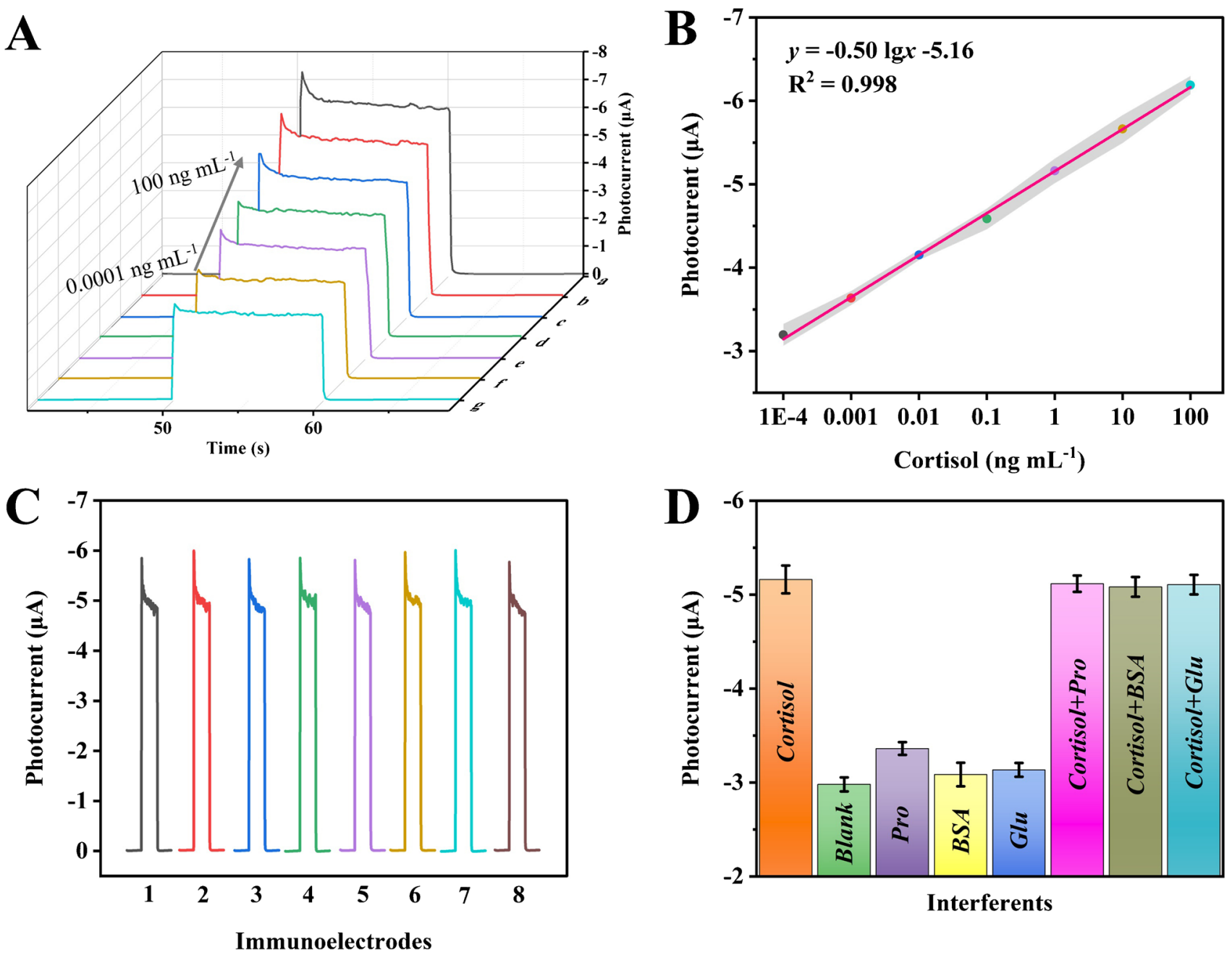

Interferents

Fig. 4 A Photocurrent response of the prepared PEC sensor toward target cortisol with different concentrations, B corresponding calibration curve within the range of $0.0001-100 \mathrm{ng} / \mathrm{mL}, \mathbf{C}$ reproducibility of the PEC sensor, and $\mathbf{D}$ selectivity test of the PEC sensor for cortisol detection

of cortisol concentration within the dynamic range of $0.0001-100 \mathrm{ng} \mathrm{mL}^{-1}$. The linear regression equation was $y=-0.50 \log \mathrm{x}+4.67\left(R^{2}=0.998\right)$ with a limit of detection (LOD) of $0.06 \mathrm{pg} \mathrm{mL}^{-1}$. Compared to other immunoassays, as seen from Table S1, our assay offered a wider detection range and a lower detection limit. To investigate the reproducibility of the method, eight independent measurements of $1 \mathrm{ng} \mathrm{mL}{ }^{-1}$ cortisol were performed in Fig. 4C, respectively, and the RSD values of the results were $1.20 \%$, indicating that the method had good reproducibility. Furthermore, the selectivity of this PEC immunosensor toward cortisol $\left(1 \mathrm{ng} \mathrm{mL}^{-1}\right)$ was also evaluated by non-target analytes, including glucose (Glu), progesterone (Pro), and BSA, and the result is shown in Fig. 4D. The system had little response to a high concentration $\left(100 \mathrm{ng} \mathrm{mL}^{-1}\right)$ of non-target analytes. On the contrary, the photocurrent signal of the mixture containing $\left(1 \mathrm{ng} \mathrm{mL}{ }^{-1}\right)$ cortisol and the non-target analyte resembled that of cortisol alone, suggesting that the PEC biosensor was extremely specific for cortisol.

\section{Detection of real samples}

To further evaluate the potential application and feasibility of Ag NCs-labelled PEC immunosensor, we examined cortisol in human saliva samples, which range from 0.6 to $12.85 \mathrm{ng} \mathrm{mL}^{-1}$ in healthy people [42, 43]. Firstly, saliva from four volunteers was collected twice in the morning and afternoon and detected by this method and ELISA after certain sample treatment (see ESM for saliva collection in details). As shown in Table 1, the detection results of the prepared PEC biosensor were close to that of ELISA. Importantly, all Student's $t$-test values of these samples were under $t_{\text {crit }}\left(t_{\text {crit }}[0.05,4]=2.78\right)$, indicating excellent reliability of this system. Surprisingly, we found vaguely that cortisol levels in the morning slightly more than in the afternoon, 
Table 1 Comparison of the measured results between the as-prepared PEC biosensor and the human salivary cortisol ELISA kit

\begin{tabular}{|c|c|c|c|c|}
\hline \multirow[t]{2}{*}{ Time } & \multirow[t]{2}{*}{ Sample no } & \multicolumn{2}{|c|}{$\begin{array}{l}\text { Method; level (mean } \pm \mathrm{SD}, \mathrm{ng} \\
\left.\mathrm{ml}^{-1}, n=3\right)\end{array}$} & \multirow[t]{2}{*}{$t_{\mathrm{exp}}$} \\
\hline & & PEC immunoassay & ELISA kit & \\
\hline \multirow[t]{4}{*}{ Morning } & 1 & $1.05 \pm 0.09$ & $0.90 \pm 0.06$ & 2.37 \\
\hline & 2 & $2.91 \pm 0.11$ & $2.81 \pm 0.16$ & 0.90 \\
\hline & 3 & $1.10 \pm 0.02$ & $0.98 \pm 0.11$ & 1.98 \\
\hline & 4 & $10.43 \pm 0.43$ & $9.36 \pm 0.75$ & 2.15 \\
\hline \multirow[t]{4}{*}{ Afternoon } & 1 & $1.06 \pm 0.06$ & $0.97 \pm 0.12$ & 1.13 \\
\hline & 2 & $2.39 \pm 0.15$ & $2.09 \pm 0.16$ & 2.30 \\
\hline & 3 & $0.97 \pm 0.05$ & $0.98 \pm 0.10$ & 0.20 \\
\hline & 4 & $9.35 \pm 0.15$ & $9.20 \pm 0.28$ & 0.81 \\
\hline
\end{tabular}

suggesting that people secreted higher cortisol in the morning, consistent with previous reports.

\section{Conclusions}

In summary, we have developed a signal-on photoelectrochemical immunoassay for salivary cortisol based on silver nanoclusters-triggered ion-exchange reaction with $\mathrm{CdS}$ quantum dots. As markers of detection antibody in competitive immunocomplexes, $\mathrm{Ag}$ NCs released thousands of $\mathrm{Ag}^{+}$ ions with acidic conditions, and $\mathrm{Ag}^{+}$ions were transferred to the PEC electrode for ion-exchange reaction with $\mathrm{CdS}$ QDs, decreasing the photocurrent and achieving signal-on type competitive immunoassay, which was suitable for the detection of small molecules. Due to the dual signal amplification of ion exchange and silver nanoclusters, the limit of detection for photoelectrochemical detection of cortisol is as low as $0.06 \mathrm{pg} \mathrm{mL}^{-1}$. The highlights of our strategy lie in the following issues: (i) the bio-recognition of this PEC sensor was performed in the liquid phase instead of on the solid-liquid interfaces, avoiding the time-consuming and complex defects of assembling photoelectric beacons, identification elements, and targets on the electrodes, boosting the stability and reproducibility; (ii) the hole-exciton capture was easily mediated by $\mathrm{Ag}_{2} \mathrm{~S}$ generated by the ion-exchange reaction between the undissolved $\mathrm{Ag}^{+}$and $\mathrm{CdS}$ QDs, thus led to significant amplification in photocurrent intensity; (iii) magnetic bead labelling could effectively separate other interferences and improve selectivity. In addition, the proposed PEC biosensor was used to test human salivary cortisol samples, displaying satisfying feasibility and accuracy compared to a commercial ELISA kit. Therefore, this work provided an alternative for the detection of pressure markers as well as other small-molecule and even PEC immunosensor applied in the complex biological system.
Supplementary Information The online version contains supplementary material available at https://doi.org/10.1007/s00216-022-03893-z.

Funding Financial support was received from the National Natural Science Foundation of China $(21864007,21605029)$ and Guizhou Provincial Natural Science Foundation (Qian Ke He Ji Chu [2020] 1Y042, [2017] 5788 Qian Ke He Platform for Talents, [2018] 5781 Qian Ke He Platform for Talents).

\section{Declarations}

The authors declare no competing interests. The use of human salivary was granted by the Ethics Committees of Guizhou University (Guizhou, China) (approval number: EAE-GZU-2021-T044). The source of human salivary was obtained from healthy volunteers, which were students in the College of Pharmacy, Guizhou University.

\section{References}

1. Zhang JS, Shuai L, Yu H, Wang ZY, Qiu MH, Lu L, et al. Acute stress, behavioural symptoms and mood states among school-age children with attention-deficit/hyperactive disorder during the COVID-19 outbreak. Asian J Psychiatry. 2020;51:102077.

2. Bo HX, Li W, Yang Y, Wang Y, Zhang QG, Cheung T, et al. Posttraumatic stress symptoms and attitude toward crisis mental health services among clinically stable patients with COVID-19 in China. Psychol Med. 2021;51:1052-3.

3. Umucu E, Lee B. Examining the impact of COVID-19 on stress and coping strategies in individuals with disabilities and chronic conditions. Rehabil Psychol. 2020;65(3):193-8.

4. Giacomello G, Scholten A, Parr MK. Current methods for stress marker detection in saliva. J Pharm Biomed Anal. 2020;191:113604.

5. Zeng HM, Liu YL, Xu ZG, Wang YJ, Chai YQ, Yuan R, et al. Construction of a Z-scheme $\mathrm{g}-\mathrm{C}_{3} \mathrm{~N}_{4} / \mathrm{Ag} / \mathrm{AgI}$ heterojunction for highly selective photoelectrochemical detection of hydrogen sulfide. Chem Commun. 2019;55:11940-3.

6. Leng DQ, Xu R, Liu L, Wang H, Li JS, Ren X, et al. Rationally engineered high-performance $\mathrm{BiVO}_{4} / \mathrm{Ag}_{3} \mathrm{VO}_{4} / \mathrm{SnS}_{2}$ photoelectrodes for ultrasensitive immunosensing of CYFRA21-1 based on HRP-tyramine-triggered insoluble precipitates. Microchim Acta. 2021;188:270.

7. Zeng ZY, Tang J, Zhang M, Pu SZ, Tang DP. Ultrasensitive zero-background photoelectrochemical biosensor for analysis of organophosphorus pesticide based on in situ formation of DNAtemplated $\mathrm{Ag}_{2} \mathrm{~S}$ photoactive materials. Anal Bioanal Chem. 2021;413:6279-88.

8. Shu J, Tang DP. Recent advances in photoelectrochemical sensing: from engineered photoactive materials to sensing devices and detection modes. Anal Chem. 2020;92:363-77.

9. Arvand M, Sayyar S, Hemmati S. Visible-light-driven polydopamine/CdS QDs hybrid materials with synergistic photocatalytic activity. J Electroanal Chem. 2019;848:113288.

10. Jalali M, Moakhar RS, Abdelfattah T, Filine E, Mahshid SS, Mahshid S. Nanopattern-assisted direct growth of Peony-like 3D $\mathrm{MoS}_{2} / \mathrm{Au}$ composite for nonenzymatic photoelectrochemical sensing. ACS Appl Mater Interfaces. 2020;12:7411-22.

11. Lin YX, Zhou Q, Tang DP. Dopamine-loaded liposomes for insitu amplified photoelectrochemical immunoassay of AFB1 to enhance photocurrent of $\mathrm{Mn}^{2+}$-doped $\mathrm{Zn}_{3}(\mathrm{OH})_{2} \mathrm{~V}_{2} \mathrm{O}_{7}$ nanobelts. Anal Chem. 2017;89(21):11803-10.

12. Zang Y, Lei JP, Hao Q, Ju HX. "Signal-on" photoelectrochemical sensing strategy based on target-dependent aptamer 
conformational conversion for selective detection of lead (II) ion. ACS Appl Mater Interfaces. 2014;6:15991-7.

13. Zhang JL, Gao Y, Zhang XC, Feng QS, Zhan CX, Song JL, et al. "Dual signal-on" split-type aptasensor for TNF- $\alpha$ : integrating MQDs/ZIF-8@ZnO NR arrays with MB-liposome-mediated signal amplification. Anal Chem. 2021;93:7242-9.

14. Zhao CQ, Ding SN. Perspective on signal amplification strategies and sensing protocols in photoelectrochemical immunoassay. Coord Chem Rev. 2019;391:1-14.

15. Yang LW, Zhang S, Liu XQ, Tang YF, Zhou YM, Wong DKY. Detection signal amplification strategies at nanomaterialbased photoelectrochemical biosensors. J Mater Chem B. 2020;8:7880-93.

16. Li F, Zhou YL, Yin HS, Ai SY. Recent advances on signal amplification strategies in photoelectrochemical sensing of microRNAs. Biosensors Bioelectron. 2020;166:112476.

17. Zhu JH, Wang M, Tu LH, Wang AJ, Luo XL, Mei LP, et al. Nanosheets-assembled hollow $\mathrm{CdIn}_{2} \mathrm{~S}_{4}$ microspheres-based photoelectrochemical and fluorescent dual-mode aptasensor for highly sensitive assay of $17 \beta$-estradiol based on magnetic separation and enzyme catalytic amplification. Sensors Actuators B Chem. 2021;347:130553.

18. Cai GN, Yu ZZ, Ren RR, Tang DP. Exciton-plasmon interaction between AuNPs/graphene nanohybrids and CdS quantum dots/ $\mathrm{TiO}_{2}$ for photoelectrochemical aptasensing of prostate-specific antigen. ACS Sensors. 2018;3:632-9.

19. Wang B, Mei LP, Ma Y, Xu YT, Ren SW, Cao JT, et al. Photoelectrochemical-chemical-chemical redox cycling for advanced signal amplification: proof-of-concept toward ultrasensitive photoelectrochemical bioanalysis. Anal Chem. 2018;90:12347-51.

20. Zhang XX, Xu D, Guo D, Han HX, Li DW, Ma W. Enzyme-free amplified SERS immunoassay for the ultrasensitive detection of disease biomarkers. Chem Commun. 2020;56:2933-6.

21. Lin YX, Zhou Q, Tang DP, Niessner R, Yang HH, Knopp D. Silver nanolabels-assisted ion-exchange reaction with CdTe quantum dots mediated exciton trapping for signal-on photoelectrochemical immunoassay of mycotoxins. Anal Chem. 2016;88:7858-66.

22. Li J, Li YL, Han M, Weng X, Li YR, Lu Z, et al. Superparamagnetic $\mathrm{Fe}_{3} \mathrm{O}_{4}$ nanoclusters embedded within porous $\mathrm{TiO}_{2}$ shells for photoelectrochemical sensing. ACS Appl Nano Mater. 2020;3:9151-7.

23. Zhang LJ, Luo ZB, Zeng RJ, Zhou Q, Tang DP. All-solid-state metal-mediated Z-scheme photoelectrochemical immunoassay with enhanced photoexcited charge-separation for monitoring of prostate-specific antigen. Biosens Bioelectron. 2019;134:1-7.

24. Song W, Zhang F, Song P, Zhang Z, He P, Li Y, et al. Untrasensitive photoelectrochemical sensor for microRNA detection with DNA walker amplification and cation exchange reaction. Sensors Actuators B Chem. 2021;327:128900.

25. Zhao Y, Tan L, Gao X, Jie G, Huang T. Silver nanoclustersassisted ion-exchange reaction with CdTe quantum dots for photoelectrochemical detection of adenosine by target-triggering multiple-cycle amplification strategy. Biosens Bioelectron. 2018;110:239-45.

26. Yan K, Karthick Kannan P, Doonyapisut D, Wu KB, Chung CH, Zhang JD. Advanced functional electroactive and photoactive materials for monitoring the environmental pollutants. Adv Func Mater. 2021;31:2008227.

27. Zhang N, Zhang L, Ruan YF, Zhao WW, Xu JJ, Chen HY. Quantum-dots-based photoelectrochemical bioanalysis highlighted with recent examples. Biosens Bioelectron. 2017;94:207-18.

28. Zhao WW, Xu JJ, Chen HY. Photoelectrochemical bioanalysis the state of the art. Chem Soc Rev. 2015;44:729-41.
29. Shu J, Tang DP. Current advances in quantum-dots-based photoelectrochemical immunoassays. Chem Asian J. 2017;12:2780-9.

30. Liu YX, Ma HM, Zhang Y, Pang XH, Fan DW, Wu D, et al. Visible light photoelectrochemical aptasensor for adenosine detection based on CdS/PPy/g- $\mathrm{C}_{3} \mathrm{~N}_{4}$ nanocomposites. Biosens Bioelectron. 2016;86:439-45.

31. Naya SI, Kume T, Akashi R, Fujishima M, Tada H. Red-lightdriven water splitting by $\mathrm{Au}($ core)-CdS(shell) half-cut nanoegg with heteroepitaxial junction. J Am Chem Soc. 2018;140:1251-4.

32. Zhang XP, Zhang RR, Yang AJ, Wang Q, Kong RM, Qu FL. Aptamer based photoelectrochemical determination of tetracycline using a spindle-like ZnO-CdS@ Au nanocomposite. Microchim Acta. 2017;184:4367-74.

33. Jiang M, Liu XY, Zhang MY, Wu ZJ, Zheng YJ, Liang YJ, et al. Hot-electron injection and charge carrier lifetime prolongation enhance the photoelectrochemical performance of a plasmonic CdS/Au photoanode. J Phys Chem C. 2021;125:17109-16.

34. Nair N, Sankapal BR. Cationic-exchange approach for conversion of two dimensional $\mathrm{CdS}$ to two dimensional $\mathrm{Ag}_{2} \mathrm{~S}$ nanowires with an intermediate core-shell nanostructure towards supercapacitor application. New J Chem. 2016;40:10144-52.

35. Valencia E, Cruz-Alonso M, Álvarez L, González-Iglesias H, Fernández B, Pereiro R. Fluorescent silver nanoclusters as antibody label in a competitive immunoassay for the complement factor H. Microchim Acta. 2019;186:429.

36. Xu H, Yuan HF, Yu JG, Lin S. Study on the competitive adsorption and correlational mechanism for heavy metal ions using the carboxylated magnetic iron oxide nanoparticles (MNPs-COOH) as efficient adsorbents. Appl Surf Sci. 2019;473:960-6.

37. Rawat D, Barman PB, Singh RR. Corroboration and efficacy of magneto-fluorescent ( $\mathrm{NiZnFe} / \mathrm{CdS}$ ) nanostructures prepared using differently processed core. Sci Rep. 2019;9:15138.

38. Ren SH, Liu SG, Ling Y, Li NB, Luo HQ. Fluorescence detection of melamine based on inhibiting $\mathrm{Cu}^{2+}$-induced disaggregation of red-emitting silver nanoclusters. Spectrochim Acta A Mol Biomol Spectrosc. 2018;201:112-8.

39. Cao LL, Zhang Q, Dai H, Fu YC, Li YB. Separation/concentration-signal-amplification in-one method based on electrochemical conversion of magnetic nanoparticles for electrochemical biosensing. Electroanalysis. 2018;30(3):517-24.

40. Junga H, Junga H, Kwona D, Leea K, Leea P, Kanga W, Yoonb TS, Kangc CJ, Leea HH. Resistive switching characteristics of $\mathrm{ZnO}$ nanoparticles layer-by-layer assembly based on cortisol and its antibody immune binding. J Ind Eng Chem. 2019;78:66-72.

41. Zhang HL, Wei B, Zhu L, Yu JH, Sun WJ, Xu LL. Cation exchange synthesis of $\mathrm{ZnS}-\mathrm{Ag}_{2} \mathrm{~S}$ microspheric composites with enhanced photocatalytic activity. Appl Surf Sci. 2013;270:133-8.

42. Dalirirad S, Han D, Steckl AJ. Aptamer-based lateral flow biosensor for rapid detection of salivary cortisol. ACS Omega. 2020;5(51):32890-8.

43. Reuster T, Buechler J, Winiecki P, Oehler J. Influence of reboxetine on salivary MHPG concentration and cognitive symptoms among patients with alcohol-related Korsakoff's syndrome. Neuropsychopharmacol. 2003;28:974-8.

Publisher's note Springer Nature remains neutral with regard to jurisdictional claims in published maps and institutional affiliations. 